\title{
A Prospective Study of Botulinum Toxin for Internal Anal Sphincter Hypertonicity in Children With Hirschsprung's Disease
}

\author{
By Robert K. Minkes and Jacob C. Langer \\ St Louis, Missouri
}

\begin{abstract}
Background: Internal anal sphincter hypertonicity with nonrelaxation can cause persistent constipation and obstructive symptoms in children after surgery for Hirschsprung's disease. Intractable symptoms traditionally have been treated with anal myectomy, which may be ineffective or complicated by long-term incontinence. The authors evaluated prospectively the use of intrasphincteric botulinum toxin for these patients.
\end{abstract}

Methods: Eighteen children were studied (age 1 to 13; median, 4 years). Botulinum toxin was injected (total dose 15 to $60 \mathrm{U}$ ) into 4 quadrants of the sphincter. Resting sphincter pressure was measured in 14 patients before and after injection. Ten have had 1 to 5 additional injections (total dose, 30 to $60 \mathrm{U}$ per injection).

Results: Four patients had no improvement in bowel function, 2 had improvement for less than 1 month, 7 had improvement for 1 to 6 months, and 5 had improvement more than 6 months. Nine of those with symptomatic improvement longer than 1 month had pressures measured, with a documented decrease in 8 . Five with no significant clinical improvement had pressure measurements, with a decrease in 3. There were no adverse effects associated with botulinum toxin injection. Four children had new encopresis postinjection, which was mild and resolved in each case.

Conclusions: Intrasphincteric botulinum toxin is a safe and less-invasive alternative to myectomy for symptomatic internal sphincter hypertonicity. Persistent symptoms, despite a fall in sphincter pressure, suggest a nonsphincteric etiology. Repeat injections often are necessary for recurrent symptoms.

J Pediatr Surg 35:1733-1736. Copyright (0) 2000 by W.B. Saunders Company.

INDEX WORDS: Botulinum toxin, Hirschsprung's disease, intestinal neuronal dysplasia.
$\mathbf{M}$ OST CHILDREN with Hirschsprung's disease have an excellent result after definitive pullthrough surgery. However, approximately $10 \%$ of patients have ongoing problems with constipation, encopresis, enterocolitis, and motility disturbances. ${ }^{1-3}$ In some children, obstructive symptoms are thought to be caused by hypertonicity of the internal anal sphincter associated with absence of the rectoanal reflex, a condition that has been termed internal sphincter achalasia. Many surgeons advocate internal sphincter myectomy or sphincterotomy ${ }^{4-6}$ for this problem. Despite their popularity, however, these procedures are not universally effective and may result in permanent injury to the sphincter.

Botulinum toxin weakens both smooth and striated muscle in a focal and transient fashion. Botulinum toxin injection into skeletal muscle has been used for many years in the treatment of strabismus and spasticity syndromes. ${ }^{7}$ More recently, botulinum toxin has been used successfully to chemically relax smooth muscle in patients with esophageal achalasia ${ }^{8}$ and chronic anal fissure, ${ }^{9}$ as an alternative to the traditional forcible dilatation, or surgical incision of the sphincter.

To determine whether botulinum toxin might be useful for treating children with Hirschsprung's disease, initial experiments in immature swine were done, the results of which showed that botulinum toxin was effective in relaxing the internal anal sphincter without any evidence of neuromuscular injury. ${ }^{10}$ Preliminary clinical experience in children with Hirschsprung's disease suggested that the technique is safe and effective in decreasing sphincter pressure, although symptoms were only relieved in 2 of the 4 children. ${ }^{11}$ Based on this information, the current study was performed to prospectively study the safety and efficacy of botulinum toxin in the management of persistent constipation and obstructive symptoms in a larger series of children with Hirschsprung's disease. In addition, we wished to determine whether clinical response to injection correlated with changes in internal sphincter pressure, and we attempted to identify clinical and manometric factors that might be predictive of symptomatic improvement.

\section{MATERIALS AND METHODS}

\section{Patient Population}

This prospective study over a 4-year period was approved by the Washington University Human Studies Committee. All patients with

From the Division of Pediatric Surgery, Department of Surgery, St Louis Children's Hospital and Washington University School of Medicine, St Louis, MO.

Address reprint requests to Jacob C. Langer, MD, Division of Pediatric General Surgery, Hospital for Sick Children, 555 University Ave, Toronto, Ontario, Canada M5G 1 X8.

Copyright $\odot 2000$ by W.B. Saunders Company

0022-3468/00/3512-0009\$03.00/0

doi:10.1053/jpsu.2000.19234 
Hirschsprung's disease who were referred for persistent obstructive symptoms after a pull-through operation were assessed by history, physical examination, barium enema, and repeat rectal biopsy (if not already performed at the referring center). Patients were considered for botulinum toxin injection if there was no evidence of mechanical obstruction or recurrent aganglionosis. Persistent obstructive symptoms included continued constipation or abdominal distension requiring aggressive medical therapy, or ongoing episodes of enterocolitis.

\section{Technique of Botulinum Toxin Injection}

All injections were done under general anesthesia in the lithotomy position. The internal sphincter was identified by palpation, and a total of 15 to 60 units of botulinum toxin (Allergan Inc, Irvine, CA) was injected through a 27-gauge needle into each of 4 quadrants at the dentate line. A volume of $0.1 \mathrm{~mL}$ was injected into each quadrant. The dose given in an individual patient was determined by the patient's weight, although the doses tended to increase during the study based on experience. Ten patients have had 1 to 5 additional injections (total dose, 30 to 60 units per injection).

\section{Anal Manometry}

Anal manometry was performed before and 1 to 3 weeks after injection in 14 patients. Manometry was performed under oral midazolam sedation $(0.75 \mathrm{mg} / \mathrm{kg})$ using a standard water-perfused system, and resting pressures were recorded as previously reported. ${ }^{11}$ At least 3 measurements were made, and the mean value was used. A decrease in sphincter pressure of at least $30 \%$ was considered significant.

\section{Assessment of Clinical Response}

Patients underwent clinical follow-up either by the author or by a local gastroenterologist, pediatric surgeon, or pediatrician. A clinical response was considered significant if the obstructive symptoms completely resolved or markedly improved for greater than 1 month. Correlation between various clinical factors and response to botulinum toxin injection were analyzed using 1-way analysis of variance, with a $P$ value of less than .05 considered significant.

\section{RESULTS}

A total of 18 patients underwent intrasphincteric botulinum toxin injection. There were 14 boys and 4 girls. Age at presentation to our center ranged from 1 to 13 years (median, 4 years). Location of transition zone was rectosigmoid in 9, descending colon in 1, transverse colon in 6, and total colon in 2 . Nine patients had a previous Soave procedure, 5 had a Duhamel, and 4 had a Swenson. Median interval between original operation and botulinum toxin injection was 4 years (range, 1 to 12 years).

Clinical response ranged from no improvement to permanent resolution of symptoms (Fig 1). Six of 18 (33\%) had either no improvement or improvement less than 1 month, and 12 of 18 (67\%) experienced improvement for greater than 1 month. Of the 6 children who did not improve, 3 were found subsequently to have intestinal neuronal dysplasia. Nine patients had more than 1 injection, either to treat recurrent symptoms or to give a higher dose after failure of the initial injection. The range of repeat injections was 1 to 5 . There was a tendency toward a lower need for repeat injections in the older children (3.6 years for

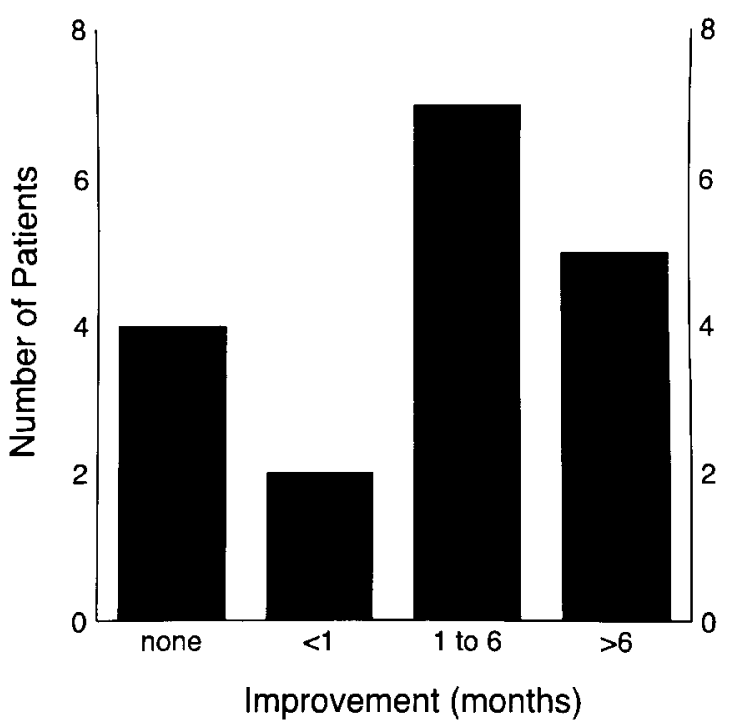

Fig 1. Duration of improvement after botulinum toxin injection in 18 patients.

those requiring repeat injections versus 5.3 years for those not requiring repeat injections), although this was not statistically significant $(P=.2)$.

Resting IAS pressures before injection of botulinum toxin ranged from 51 to $192 \mathrm{~mm} \mathrm{Hg}$. Postinjection pressures ranged from 36 to 155 . Of the 5 patients without clinical improvement who had pre- and postinjection manometry, 3 had a significant decrease in IAS pressure, and 2 did not (Fig 2). Nine of the 12 patients with symptomatic improvement had pre- and postinjection manometry, 8 of whom had a significant decrease in IAS pressure (Fig 2).

A number of factors were evaluated as possible predictors for clinical response to botulinum toxin injection. There was no statistical correlation between clinical response and age at diagnosis of Hirschsprung disease, age at time of injection, history of a previous colostomy or previous myectomy, type of pull-through procedure, or preinjection IAS pressure. However, patients with more distal transition zones appeared to be more likely to respond to botulinum toxin injection than those with longer segment disease (Fig 3).

There were no patients who had infectious or bleeding complications from botulinum toxin injection. Several patients complained of transient anal or abdominal pain, all of which resolved within several hours. New encopresis developed in 4 patients postinjection, 2 of whom had a previous myectomy. In each of these cases the incontinence was termed as mild and resolved within 3 weeks. There were no other adverse effects associated with injection. 


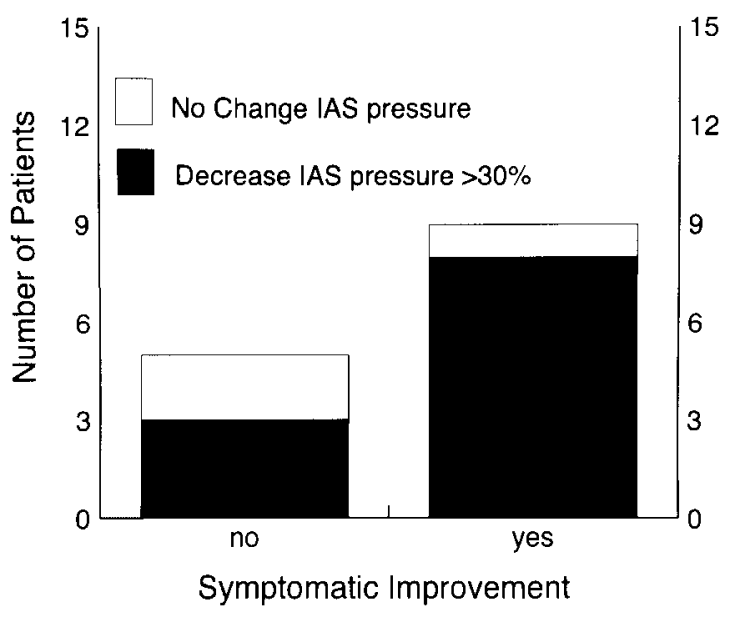

Fig 2. Relationship between clinical response and change in internal anal sphincter (IAS) pressure. Symptomatic improvement was defined as clinical improvement for greater than 1 month.

\section{DISCUSSION}

Botulinum toxin acts by blocking acetylcholine release at the neuromuscular junction, and produces a flaccid paralysis, which resolves within 3 to 6 months. Botulinum toxin has been used therapeutically for many years to treat skeletal muscle conditions, which may be associated with hyperfunctioning cholinergic nerve terminals. ${ }^{12,13}$ It has been shown to be safe in both adults and children and has been used successfully for relaxation of skeletal muscle spasticity as well as in the smooth muscle of the lower esophageal and internal anal sphincters. This prospective study extends the previously published experience using botulinum toxin for internal anal sphincter hypertonicity associated with Hirschsprung disease ${ }^{11}$ and shows that botulinum toxin injection into the internal anal sphincter is a safe and less invasive alternative to myectomy for the management of these patients.

It has been noted by a number of investigators that short segment Hirschsprung's disease and long segment aganglionosis may behave in different ways. These observations include a higher incidence of enterocolitis, absence of hypertrophic nerves, and a higher incidence of known gene mutations ${ }^{14,15}$ in patients with extensive aganglionosis. Our finding that patients with shorter segment disease were more likely to respond to botulinum toxin therapy than those with more proximal transition zones documents another difference between these 2 groups and suggests that obstructive symptoms in long segment cases may be caused by intestinal neuronal dysplasia or other motility disorders. No other factors, including the preinjection resting IAS pressure, were found to be predictive of response to botulinum toxin.

The duration of response to injection was variable, and half of the patients have undergone multiple injections.
Repeat treatment is indicated for 2 groups of patients: (1) those with no clinical improvement who have not had a decrease in IAS pressure (ie, the injection did not relax the sphincter, and a higher dose of botulinum toxin may be necessary) and (2) patients with a good clinical response who have recurrent symptoms when the botulinum toxin wears off. Repeated injections of botulinum toxin have been shown to be safe and effective in other clinical settings, ${ }^{7-13,16}$ and we have not observed any adverse effects. Late results and potential adverse effects of multiple injections will await longer follow-up.

In addition to its therapeutic value, botulinum toxin injection also may provide useful diagnostic information. Patients who respond to botulinum toxin may be appropriate candidates for myectomy. However, all patients in our series, when given the option, have selected repeat injection over myectomy. However, a patient with no clinical response associated with a documented decrease in IAS pressure after injection is likely to have a nonsphincteric etiology for their obstructive symptoms. This subset of patients should be evaluated for other conditions, such as intestinal neuronal dysplasia or stoolholding behavior. We have found laparoscopy to be a useful approach for ruling out extraluminal causes of obstruction, such as adhesions, and to perform multiple full-thickness biopsies of the colon and small intestine for histologic examination and cholinesterase staining. ${ }^{17}$ Of the 6 patients in our series who did not improve after injection, 3 subsequently were found to have intestinal neuronal dysplasia using this technique.

In our series, incontinence was more likely to occur in patients who did not have normal continence before botulinum toxin therapy and in those patients who had a prior myectomy. However, all patients who were continent before injection regained continence within several

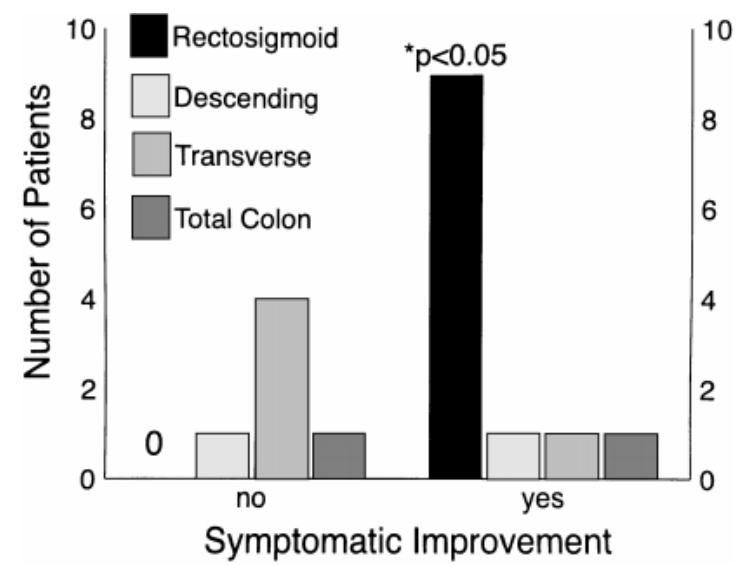

Fig 3. Relationship between length of aganglionic segment and duration of response after botulinum toxin injection. Patients with shorter segment disease had a better response to botulinum toxin. (*) One-way analysis of variance. 
weeks, confirming the reversibility of the effect. It has been reported that antibodies against the toxin can develop and diminish or prevent a therapeutic response. ${ }^{13}$ There also is experimental evidence that natural resistance to botulinum exists. ${ }^{18}$ Use of the smallest possible dose and longer treatment intervals may reduce the incidence of adverse effects of the toxin as well as the antibody-mediated resistance. ${ }^{13}$ However, we have not yet had any patients in whom tolerance developed after an initial positive response.

Botulinum toxin is a safe and effective tool that provides important diagnostic information in children with Hirschsprung's disease who have obstructive symptoms after pull-through surgery. It also provides a therapeutic alternative to myectomy or sphincterotomy in the majority of these patients.

\section{REFERENCES}

1. Tariq GM, Brereton RJ, Wright VM: Complications of endorectal pull-through for Hirschsprung's disease. J Pediatr Surg 26:1202-1206, 1991

2. Marty TL, Seo T, Matlak ME, et al: Gastrointestinal function after surgical correction of Hirschsprung's disease: Long-term follow-up in 135 patients. J Pediatr Surg 30:655-658, 1995

3. Heij HA, de Vries X, Bremer I, et al: Long-term anorectal function after Duhamel operation for Hirschsprung's disease. J Pediatr Surg 30:430-432, 1995

4. Kimura K, Inomata Y, Soper RT: Posterior sagittal rectal myectomy for persistent rectal achalasia after the Soave procedure for Hirschsprung's disease. J Pediatr Surg 28:1200-1201, 1993

5. Blair GK, Murphy JJ, Fraser GC: Internal sphincterotomy in post-pull-through Hirschsprung's disease. J Pediatr Surg 31:843-845, 1996

6. Abbas Banani S, Forootan $\mathrm{H}$ : Role of anorectal myectomy after failed endorectal pull-through in Hirschsprung's disease. J Pediatr Surg 29:1307-1309, 1994

7. Jankovic J, Brin MF: Therapeutic uses of botulinum toxin. N Engl J Med 324:1186-1194, 1991

8. Pasricha PJ, Rai R, Ravich WJ, et al: Botulinum toxin for achalasia: Long-term outcome and predictors of response. Gastroenterol 110:1410-1415, 1996

9. Gui D, Cassetta E, Anastasio G, et al: Botulinum toxin for chronic anal fissure. Lancet 344:1127-1128, 1994
10. Langer JC, Birnbaum EE, Schmidt RE: Histology and function of the internal anal sphincter after injection of botulinum toxin. J Surg Res 73:113-136, 1997

11. Langer JC, Birnbaum EE: Preliminary experience with intrasphincteric botulinum toxin for persistent constipation after a pullthrough for Hirschsprung's disease. J Pediatr Surg 32:1059-1062, 1997

12. Jankovic J, Brin MF: Botulinum toxin: Historical perspective and potential new indications. Muscle \& Nerve Supplement 6:S129-45, 1997

13. Brin MF: Botulinum toxin: Chemistry, pharmacology, toxicity, and immunology. Muscle \& Nerve Supplement 6:S146-168, 1997

14. Jasonni V, Martucciello G: Total colonic aganglionosis. Semin Pediatr Surg 7:174-180, 1998

15. Kusafuka T, Puri P: Genetic aspects of Hirschsprung's disease. Semin Pediatr Surg 7:148-155, 1998

16. Pasricha PJ, Ravich WJ, Hendrix TR, et al: Intrasphincteric botulinum toxin for the treatment of achalasia. N Engl J Med 322:774778, 1995

17. Langer JC, Mazziottti MV: Laparoscopic full thickness intestinal biopsies in children. Gastroenterology 116:A560, 1999

18. Coffield JA, Bakry NM, Maksymowych AB, et al: Characterization of a vertebrate neuromuscular junction that demonstrates selective resistance to botulinum toxin. J Pharmacol Exp Ther 289:15091516, 1999 